\title{
Are We Missing Osteoporosis-Related Vertebral Fractures in Men?
}

\author{
Mir Sadat-Ali ${ }^{1,2}$, Abid Hussain Gullenpet ${ }^{3}$, Haifa A. Al-Turki ${ }^{1,4}$, Tamar W. AbdulRahman ${ }^{3}$, \\ Abdulmohsen $\mathrm{H}$. Al-Elq ${ }^{1,5}$, Mohammed Quamar Azzam², Hadia Al-Shammary ${ }^{3}$, \\ Abdallah S. Al-Omran², Abdallah A. Al-Othman²
'Osteoporosis Research Group, College of Medicine, University of Dammam, Saudi Arabia
${ }^{2}$ Department of Orthopaedic Surgery, King Fahd Hospital of the University, University of Dammam College of Medicine, Al Khobar, Saudi Arabia ${ }^{3}$ Department of Radiology, King Fahd Hospital of the University, University of Dammam College of Medicine, Al Khobar, Saudi Arabia ${ }^{4}$ Department of Obstetrics and Gynaecology, King Fahd Hospital of the University, University of Dammam College of Medicine, Al Khobar, Saudi Arabia ${ }^{5}$ Department of Internal Medicine, King Fahd Hospital of the University, University of Dammam College of Medicine, Al Khobar, Saudi Arabia

\section{Study Design: Retrospective study.}

Purpose: To assess the prevalence of osteoporosis related spinal fractures among Saudi Arabian males.

Overview of Literature: Vertebral fractures are the most common complication of osteoporosis and is the first sign in both sexes and only 25 to $30 \%$ of radiographically observed vertebral deformities are recognized.

Methods: We analyzed the chest radiographs of consecutive Saudi Arabian men $\geq 50$ years and who visited the emergency room of King Fahd University Hospital, Al Khobar, Saudi Arabia for a period of 12 months between November 1, 2007 and October 31, 2008. The site and type of fractures were classified as per the semi-quantitative technique. The other data retrieved from the medical records of patients included medications and clinical investigations for osteoporosis.

Results: Nine hundred seventy chest radiographs were performed during the study period and 876 radiographs could be analyzed. One hundred fifteen patients (13.1\%) had 157 fractures. The mean age was $67.85 \pm 10.1$ years. There was more than one fracture in 21 patients (18.2\%). The majority of fractures ( $n=102,64.9 \%$ ) were observed in thoracic spine. Seventyone (45.2\%) fractures were classified as mild, 54 (34.4\%) were moderate and 32 (20.4\%) were severe. For 26 (22.6\%) patients, the report of the radiologist highlighted the fracture.

Conclusions: Saudi Arabian males with osteoporosis continue to be missed despite the high prevalence osteoporosis leading to vertebral fractures. We believe it is important for physicians to identify vertebral fractures early and treat then appropriately before an extremity fracture occurs with high mortality.

Key Words: Male osteoporosis, Vertebral fractures, Saudi Arabians

\section{Introduction}

Osteoporosis is becoming a major preventable disease with high morbidity and mortality due to its related fractures. It was previously believed that osteoporosis in males was rare and so the complications that postmenopausal women suffer are not seen in men. In the United States, the

Received Jun 8, 2010; 1st Revised Jul 4, 2010; 2nd Revised Aug 3, 2010; Accepted Aug 5, 2010

Corresponding author: Mir Sadat-Ali, MS, FRCS

Department of Orthopaedic Surgery, King Fahd University Hospital, PO BOX 40071, Al Khobar 31952, Saudi Arabia

Tel: +966505848281, Fax: +96638820887, E-mail: drsadat@hotmail.com 
ratio of females with osteoporosis to males with osteoprosis is $3: 1$ (30 million women and 10 million men) for the people aged 50 years or older [1]. The incidence of male osteoporosis, as reported in 1997, was between 5-8\% in US men [2], and this jumped to $18-35 \%$ depending on the different diagnostic reference that is used [3]. Sadat-Ali and AlElq [4] reported the prevalence of osteopenia and osteoporosis in Saudi males to be around $35 \%$ and $37.4 \%$, and similar findings were found in the Riyadh region [5].

Atraumatic spinal fracture due to osteoporosis is the most frequent type of osteoporotic fracture [6] and this goes unnoticed till an extremity fractures occurs in 1 of 4 men older than 60 years [7]. Hip fracture is particularly disabling and it is associated with a risk of mortality and prolonged morbidity [8,9]. Al-Omran and Sadat-Ali [10] reported that only $20 \%$ of the post hip fracture patients go back to the pre-fracture status. Although there are many risk factors for secondary osteoporosis in men, making an early diagnosis and administering proper treatment remain neglected. One of the ways to assess whether a proper diagnosis of male osteoporosis has been done is by reviewing the diagnosis of spinal fracture as this could be the first red flag for an impending hip fracture. This study is carried out to determine the prevalence of vertebral fractures within a 12month period and to determine what percentage of the fractures were missed.

\section{Materials and Methods}

After the approval of the institutional research and ethical committee, this study was carried out at King Fahd University Hospital, AlKhobar. The medical records of all the Saudi male patients $\geq 50$ years old and who had a chest radiograph taken in the emergency room during the 12 months between November 1, 2007 and October 31, 2008 were obtained. The information on age, the investigations, the medications and especially the antiresorptives, vitamin $\mathrm{D}$ and calcium was gathered. The medical records were reviewed for the weight, height, smoking habits, and physical activities. A sedentary life style was defined as minimal physical activity amounting to $<15$ minutes of daily walking within the home. Radiographs (the antero-posterior and lateral views) that showed the thoraco-lumbar junction were retrieved from the Picture Archiving and Communication System (PACS, Siemens AG, Erlangen, Germany), and these were reviewed independently reviewed by the authors AHG, TM and HS, and they were later jointly reviewed.
The site of the fractures and the type were classified as mild, moderate or severe as per the semi-quantitative technique according to Genant et al. [11]. In the mild fractures there is collapse/wedging of $\leq 25 \%$ of the vertebral body, in the moderate fractures there is a collapse between 26$40 \%$ and severe fractures have $\geq 41 \%$ collapse of the body. Patients with the diagnosis of a malignancy or a connective tissue disorder and those on steroids were excluded from the analysis. The Ulticare patient care system was checked for the report of the chest radiographs and subsequent fractures. All the data was entered in the data base and it was analyzed using SPSS ver. 14 (SPSS Inc., Chicago, IL, USA).

\section{Results}

During the study period, 970 chest radiographs were performed and 876 radiographs could be analyzed. One hundred fifteen patients $(13.1 \%)$ had 157 fractures. The mean age was $67.85 \pm 10.1$ years. Table 1 shows the demographic data of the patients with vertebral fractures. In 21 patients $(18.2 \%)$ there was more than one fracture (Table 2). The majority of the fractures $(n=102,64.9 \%)$ were observed in the thoracic spine and specifically in the thoracic 7 th, 9th and 10th vertebral bodies. Seventy-one (45.2\%) fractures were classified as mild, 54 (34.4\%) were moderate and 32

Table 1. Demographic data of 115 men with fractures

\begin{tabular}{lcc}
\hline \hline Parameter & Mean \pm SD & Range \\
\hline Age (yr) & $67.8 \pm 10.1$ & $53-88$ \\
BMI (M/kg²) & & \\
$\quad \leq 25$ & $30(26)$ & \\
$\quad 26-30$ & $73(63.5)$ & \\
$\quad \geq 31$ & $12(10.5)$ & \\
Smokers & $(52)$ & \\
SDL & $(79)$ & \\
Haemoglobin (g/\%) & $12.6 \pm 3.1$ & $9.9-14.6$ \\
ESR & $35.4 \pm 19$ & $3.94-4.62$ \\
FBS (mg/dl) & $118 \pm 25$ & $8.9-10.2$ \\
Albumin (g/dl) & $4.1 \pm 0.7$ & $3.1-4.4$ \\
Calcium (mg/dl) & $9.7 \pm 0.5$ & $54-304$ \\
Phosphorus (mg/dl) & $3.4 \pm 0.7$ & \\
AP (I/U) & $122 \pm 39$ & \\
\hline
\end{tabular}

Values are presented as number $(\%)$.

SD: Standard deviation, BMI: Body mass index, SDL: Sedentary life style (minimal physical activity amounting daily to < 10 min of walking within the house), ESR: Erythrocyte sedimentation rate, FBS: Fasting blood sugar, AP: Alkaline phosphatise. 
Table 2. Age group of 115 patients with 157 vertebral fractures

\begin{tabular}{cccc}
\hline \hline Age range (yr) & Single fracture & $\geq 2$ fractures & Total \\
\hline$\leq 59$ & 27 & 9 & 36 \\
$60-69$ & 32 & 13 & 45 \\
$70-79$ & 35 & 15 & 50 \\
$80-88$ & 21 & 5 & 26 \\
\hline
\end{tabular}

(20.4\%) were severe. The report of the radiologist highlighted the fracture for 26 (22.6\%) patients. Eight patients $(6.9 \%)$ had a bone mineral density scan was ordered and none of the men who had spinal fracture were on antiresorptive therapy.

Sixteen (13.9\%) patients with vertebral fractures presented with another extremity fracture. There were 9 femoral fractures, 2 fractures of the neck of the humerus, 3 Colles fractures and 2 diaphyseal tibial fractures. Table 3 shows the primary disease for which the patients were attending the hospital.

\section{Discussion}

This study shows that the prevalence of silent vertebral fractures due to osteoporosis in male Saudi Arabs in the eastern province was $13.1 \%$. It was found that $5 \%$ of men in the USA suffer from vertebral fractures due to osteoporosis [12], but another report from Mexico puts this figure at around $13 \%$ [13]. We have observed that the majority of patients were hospitalized for their primary disease and with a fracture that was missed, and the opportunity to treat the fracture was also missed. Even though the prevalence of osteoporosis vertebral fractures in men is lower than that in women (13\% vs. $25 \%$ ) [14-16], the morbidity seen in our patients was thought to be high because of the smoking habits of men in general and most of our patients suffered from cardio-pulmonary diseases. Osteoporosis in men has recently received much attention due to the increased mortality after hip fractures as compared to that in women $[17,18]$. Still, physicians neglect to investigate vertebral fractures in men and these occur much earlier than the hip fractures. The simple way of diagnose silent vertebral fractures is either by mean of serial height measurements in males attending hospital/clinics [19] or by plain lateral radiographs [20].

The diagnosis of osteoporosis related vertebral fractures is still a contentious issue. It was reported that missed vertebral fractures by radiologists and physicians is universal
Table 3. Primary department of care of patients with fractures

\begin{tabular}{lc}
\hline \hline Speciality & No. of patients $(\%)$ \\
\hline Cardiology & $45(39.1)$ \\
Pulmonary & $21(18.3)$ \\
Nephrology & $11(9.6)$ \\
Internal Medicine & $13(11.3)$ \\
Opthalmology & $6(5.2)$ \\
General Surgery & $4(3.5)$ \\
Psychiatry & $5(4.3)$ \\
First time visitor & $10(8.7)$ \\
\hline
\end{tabular}

except that the incidence varies between $29-45 \%$, with the lowest incidence being in the Europe and the highest in North and South America [21]. In this study 22.6\% of patients had a report by a radiologist that indicated a vertebral fracture, but the physicians still failed to take notice. There are a few possible explanations why such a large number $(81 \%)$ of the fractures were missed by the radiologists. First, the radiologists were concentrating on the organs in the chest cavity and they failed to recognize the skeletal structures, and second the radiographs were reviewed and reported on by staff with different levels of experience, and third the learning curve for semi-quantitative morphometry is very high, and last osteoporosis and osteoporosis-related fractures in men were never on the agenda for the diagnosis, and these maladies were left untreated by the physicians. None of the patients had any treatment for osteoporosis and this resulted in extremity fractures in 16 patients.

This study has some limitations like all retrospective studies. The second time around the radiologists were more focused to diagnose vertebral fractures rather than looking at the chest radiograph as a whole. Second, there might have been patients who did not have chest radiographs and who had osteoporosis related vertebral fractures. Last, the prevalence of fractures in this study does not represent the prevalence of osteoporosis related vertebral fractures for the whole country.

In conclusion, this study shows that osteoporosis related vertebral fractures in Saudi males are not uncommon. We believe there is need to increase the awareness among radiologists and physicians that male osteoporosis is not rare and males can suffer fractures the same as postmenopausal women. This is important because the Saudi Arabian population is shifting towards an elderly age and this may cause a further increase the incidence of osteoporosis. Physicians have to act now to diagnose osteoporosis related vertebral 
fractures early, and physicians need to investigate and treat osteoporosis properly so that extremity fractures can be prevented.

\section{Conclusions}

Osteoporosis related vertebral fractures are common among Saudi Arabian males and missed by radiologists and clinicians. We believe more is needed to prevent this happening so that extremity fractures could be prevented by early diagnosis and appropriate treatment of osteoporosis.

\section{REFERENCES}

1. Gronholz MJ. Prevention, diagnosis, and management of osteoporosis-related fracture: a multifactoral osteopathic approach. J Am Osteopath Assoc 2008;108:575-85.

2. Looker AC, Orwoll ES, Johnston CC Jr, et al. Prevalence of low femoral bone density in older U.S. adults from NHANES III. J Bone Miner Res 1997;12:1761-8.

3. Richy F, Gourlay ML, Garrett J, Hanson L, Reginster JY. Osteoporosis prevalence in men varies by the normative reference. J Clin Densitom 2004;7:127-33.

4. Sadat-Ali M, AlElq A. Osteoporosis among male Saudi Arabs: a pilot study. Ann Saudi Med 2006;26:450-4.

5. El-Desouki MI, Sulimani RA. High prevalence of osteoporosis in Saudi men. Saudi Med J 2007;28:774-7.

6. Cauley JA, Hochberg MC, Lui LY, et al. Long-term risk of incident vertebral fractures. JAMA 2007;298:2761-7.

7. Gruntmanis U. Male osteoporosis: deadly, but ignored. Am J Med Sci 2007;333:85-92.

8. Kanis JA. Diagnosis of osteoporosis and assessment of fracture risk. Lancet 2002;359:1929-36.

9. Grisso JA, Kelsey JL, Strom BL, et al. Risk factors for falls as a cause of hip fracture in women. The Northeast Hip Fracture Study Group. N Engl J Med 1991;324:1326-31.

10. Al-Omran A, Sadat-Ali M. Is early mortality related to timing of surgery after fracture femur in the elderly? Saudi
Med J 2006;27:507-10.

11. Genant HK, Wu CY, van Kuijk C, Nevitt MC. Vertebral fracture assessment using a semiquantitative technique. J Bone Miner Res 1993;8:1137-48.

12. Osteoporosis in men [Internet]. Bethesda (MD): National Institutes of Health Osteoporosis and Related Bone Diseases-National Resource Center [Cited 2010 Mar 20]. Available from: http://www.niams.nih.gov/Health_Info/ Bone/Osteoporosis/men.asp.

13. Clark P, Cons-Molina F, Deleze M, Talavera JO, Palermo L, Cummings SO. The prevalence of radiographic vertebral fractures in Mexican men. Osteoporos Int 2010;21:1523-8.

14. Majumdar SR, Kim N, Colman I, et al. Incidental vertebral fractures discovered with chest radiography in the emergency department: prevalence, recognition, and osteoporosis management in a cohort of elderly patients. Arch Intern Med 2005;165:905-9.

15. Grados F, Marcelli C, Dargent-Molina P, et al. Prevalence of vertebral fractures in French women older than 75 years from the EPIDOS study. Bone 2004;34:362-7.

16. Díaz López JB, Naves Díaz M, Gómez Alonso C, Ferná ndez Martín JL, Rodríguez Rebollar A, Cannata Andía JB. Prevalence of vertebral fracture in population older than 50 years in Asturias (Spain) defined following different radiological criteria. Med Clin (Barc) 2000;115:326-31.

17. Gennari L, Bilezikian JP. Osteoporosis in men. Endocrinol Metab Clin North Am 2007;36:399-419.

18. Bass E, French DD, Bradham DD, Rubenstein LZ. Riskadjusted mortality rates of elderly veterans with hip fractures. Ann Epidemiol 2007;17:514-9.

19. Vestergaard P, Rejnmark L, Mosekilde L. Has mortality after a hip fracture increased? J Am Geriatr Soc 2007;55: 1720-6.

20. Campion JM, Maricic MJ. Osteoporosis in men. Am Fam Physician 2003;67:1521-6.

21. Delmas PD, van de Langerijt L, Watts NB, et al. Underdiagnosis of vertebral fractures is a worldwide problem: the IMPACT study. J Bone Miner Res 2005;20:557-63. 